\title{
Review of Naturaleza creativa
}

\section{GEOFFREY WOOLLARD}

Department of Medical Biophysics, University of Toronto, Toronto, Canada geoff.woollard@mail.utoronto.ca

ORCID: 0000-0001-5440-4228

\section{JOHN G. BRUNGARDT}

Instituto de Filosofía, Pontificia Universidad Católica de Chile, Santiago, Chile jobrungardt@uc.cl

ORCID: 0000-0003-4989-145X

\begin{abstract}
The short monograph Creative Nature (Francisco Javier Novo, Rubén Pereda, and Javier Sánchez -Cañizares. 2018. Naturaleza Creativa. Madrid: Rialp. ISBN: 978-84321-4916-0.196 pp. Paperback, €14.25) is a welcome contribution to the philosophy of nature that arose from interdisciplinary conversations between authors who are both up-to-date in the scientific literature and deeply grounded in the western intellectual tradition. The authors draw from modern physics, biochemistry, evolutionary biology, developmental biology and ecology to argue that nature is creative in the sense that an "open future" of our evolving world lies ahead. In this review essay, divided into two parts, we offer a chapter-by-chapter summary covering Nature, Life, Change, Limits, Functions and Creativity. In conclusion, we offer some pedagogical possibilities. The second part proposes certain points for deeper reflection.
\end{abstract}

Keywords: philosophy of nature; evolution; relationship to nature; boundary questions; form and finality. 


\section{Introduction}

Ideally the physicist should be allowed to elucidate his own universe up to a point, and then hand it over to the philosopher to ascertain its exact status in relation to a wider outlook. But in practice we have not sufficient confidence in one another, and we both make raids over the border to suggest all sorts of ways in which the other fellow may be deceiving himself and us.

- Sir Arthur Eddington, "Physics and Philosophy,” Philosophy 8.29 (1933): 30

It is increasingly a commonplace no longer believed that the realms of philosophy and science ought to have little to do with each other. While generations of philosophers and scientists in the late 19th- and 20th-centuries were trained into a cultural antipathy towards the other discipline, few achieved true education or mastery without a deep appreciation and facility in the concepts of their neighbor's territory. In today's scholarly literature, one can point to Mariano Artigas, Benedict Ashley, G.F.R. Ellis, or Nancey Murphy, among many others, as instances of philosophers and scientists intent upon bridging these domains in a meaningful and profound way. ${ }^{1}$ The short monograph Naturaleza creativa (Creative Nature ${ }^{2}$ ) enters into this line of holistic thinking in the philosophy of nature by being itself a fruit of many interdisciplinary conversations between its three authors. It serves as a pedagogy of disciplined wonder about nature not as a realm disenchanted by modernity's fragmentation of knowledge and tradition but as a marvelous order characterized by intelligible novelty and innovation of form. The universe, the authors claim, is "presented by current science as a dynamic system whose basic characteristic is creativity," that is, "a process still incomplete and, consequently, open to the future" ${ }^{3,4}$ Nonetheless,

1 See Artigas 2001, Artigas 2006, Ashley 2006, Ellis 2016, Murphy and Brown 2007, Murphy and Stoeger, 2007.

2 The emphasis here is nature as creative-the creativity inherent in natural processes. Nor should the similar name imply that the authors defend older theories of "creative nature" like Herbert Spencer or the "creative evolution" of Henri Bergson.

3 All translations of the book under review are our own.

4 Novo et al. 2018, 13, 179. 
Nature is no Heraclitean flux. ${ }^{5}$ Rather, its development and evolution is characterized by a dynamic, chaotic, and intelligible unfolding of order that constitutes its innovation on cosmic, geological and evolutionary timescales. An attentiveness to the unifying themes shared by the experimental sciences as well as the perennial relevance of formal causality and finality in nature makes this book particularly attractive for interdisciplinary education in philosophy and the sciences. Students guided through this book, perhaps in a series of seminars co-led by a scientist and a philosopher, would be well served. In this review essay we first summarize the book and then offer points for deeper reflection that were the fruit of our own interdisciplinary conversations.

\section{Chapter Review}

The book comprises six chapters with an introduction and epilogue. Each chapter ends with a lapidary summary and unfolds with an amiable tone that guides the reader by the hand. They read like a "best of" report of the conversations held while the authors wrote the book, and makes one wish to engage in similar discussions with one's colleagues.

\subsection{Brief Outline and Summary}

The introduction frames the goal of the book in terms of recapturing a true wonder at nature that "loves to hide" in Heraclitus's memorable phrase. To inspire such wonder, the authors open the first chapter with "a walk in the park" where they draw the reader's attention to the levels of clear and hidden order in the workings of nature. The subsequent chapters consider, respectively, the nature of life, change, limitation, function, and creativity,

5 We note that one of its authors contributes to a recent article discussing the difficulty of a Heraclitean conception of nature and its encounter with the "meta-law dilemma." See Barrett and Sánchez Cañizares 2018, in response to the philosophy of nature defended by the philosopher and scientist (respectively) Roberto Mangabeira Unger and Lee Smolin in their book The Singular Universe and the Reality of Time: A Proposal in Natural Philosophy (Unger and Smolin 2014). 
each striving in a philosophically sober and scientifically astute way to deepen our initial appreciation of our walk through the park. This tour aims, as our authors note in the epilogue, to provide us with what one could call an introduction to the philosophy of nature: "To understand Nature as a great system of complex and dynamic systems, modern science no longer sees the Universe as a 'machine' moved solely by cold, deterministic interactions, but rather as an immense network of relations." "The book thus provides a contemporary gloss on the medieval notion that the universe is essentially characterized as a unity of distinct yet ordered members. ${ }^{7}$

The authors set up the book by stressing the limits of our knowledge, our alienation from nature, and the loss of meaning that we experience in a postmodern environment. To regain our grasp of nature's meaning they critique the mechanistic worldview of nature that has come down to us from the era of Newton and his timeless and all-encompassing laws. Yet the corrective is not to return to a Greek vision of nature as a living being, which is often misunderstood since we are twice removed from that age. The authors gratefully receive insights from antiquity and the middle ages and incorporate them into the contemporary worldview of nature not only a pattern of numbers (cf. Stanley Jaki), but a dynamic unfolding of possibilities, which are conditioned by past events. Their fresh perspective combines the themes of complexity, systems thinking and ecological evolutionary development (Eco-Evo-Devo) with themes of emergence and openness (cf. Stuart Kauffman).

The authors first look at the order in nature, reflecting on contributions from contemporary physics (Chapter One: Nature). They then look at what dramatically stands out in nature: life. They distinguish living entities from non-living entities thermodynamically (Chapter Two: Life) and evolutionarily (Chapter Three: Change). They connect microscopic physical order with

\footnotetext{
Novo et al. 2018, 182.

See, for instance, St. Thomas Aquinas: "The form of the universe consists in the distinction and order of its parts" (Aquinas 1918, ScG, II.39, Leon., 13: 358). By “parts” Aquinas means, firstly, kinds of being and, secondly, individual instances of those kinds. All translations of Aquinas are our own, unless indicated otherwise, and for such translations we cite the Leonine edition of Aquinas' works.
} 
evolution by discussing molecular mechanisms in a general framework of Eco-Evo-Devo. In this new and enriched evolutionary synthesis they examine what states are possible for nature and what exactly natural selection selects (Chapter Four: Limits). They distinguish "cause" from related concepts, distinguish "form" and "function" and discuss order in both general and living entities (Chapter Five: Functions). They address the ongoing debate on finality/purpose/teleology through the synthesis they have composed in the book, to which both physics and biology contribute. Lastly, with further contributions from modern physics, particularly quantum mechanics, they discuss biological/natural "laws" and several types of emergence (Chapter Six: Creativity). By the end of the book they have explained how nature is creative, leaving the book's title unambiguous and well-elaborated.

\subsection{Chapter-by-Chapter Summary}

Chapter One: Nature

The first chapter entitled "Nature" has four parts: "A Walk in the Park", "Systems", "Complexity" and "Strategies". In the first part, the authors use a casual stroll through the park as a pedagogical device for connecting our everyday experience with reflection typical of the philosophy of nature. As we walk through the park, do we experience a whole, a cosmos? When we take things apart and see how they are made, can we put them back together into a whole (Sacks 2012)? ${ }^{8}$

In "Systems" the authors look at trends in physics to understand nature as a unified totality, a dynamic interconnected system. The authors also examine themes common to all scientific disciplines, such as noise. The ancients had a more open view towards chance events than did many writers around the time of Newton who viewed nature as an optimized system ${ }^{9}$ or machine. Likewise, the authors remain open to randomness/stochasticity as

8 This dichotomy is inspired by the way Rabbi Lord Jonathan Sacks summarizes the difference between science and religion.

9 Contrast Gottfried Leibniz "le meilleur des mondes possibles" in Essais de Théodicée sur la bonté de Dieu, la liberté de l'homme et l'origine du mal in 1710 with Voltaire's 1759 crass satire Candide, ou l'Optimisme. 
an inherent aspect of nature. If our scientific education concluded at a basic level, and we did not benefit from advanced studies, we may indeed still consider ourselves obliged to hold a mechanistic worldview. However, the authors set out to show that contemporary science, now fanned out into an impressive number of specialized sub-domains, points to something beyond. This book sets out to integrate the specialized findings from a number of disciplines and not lose the forest for the trees. Creative Nature, as a work of philosophy of nature, builds and solidifies bridges between these particular disciplines by taking up their unique and complementary perspectives in a unified approach.

The third and fourth part of chapter one, "Complexity" and "Strategies", take up perspectives from physics, especially chaos theory (i.e., nonlinear dynamics), and explore themes in chemistry and biology such as long term prediction in complex (living) systems, phase transitions, interaction networks, nested levels of complexity and temporal rhythms.

How well do we really understand what makes living beings alive? We currently study living entities at many physical scales: microscopic properties of biomolecules, mechanisms of physico-chemical communication, as well as organismal and population evolutionary fitness (Bershtein et al. 2017). Despite enormous advances in science that Creative Nature builds on, it never hurts to remember that gaps remain. As our knowledge improves the gaps narrow, but this creates new gaps. For instance, when, between two species (s1 and s2) evolutionary biologists infer a 'missing link' (s1.5), there are now two missing links between s1-s1.5 and s1.5-s2 instead of one.

Creative Nature provides a welcome opportunity to refine the mental pictures that help us provisionally fill in gaps. How similar are the provisional mental pictures that bridge micro, meso and macro scales? How do we imagine the classical limit as we zoom out from an active site of a protein to an organelle, cell, tissue, organ, system and organism (cells to systems)? How do we fast forward through evolutionary time scales in our mind's eye? What intuition do we have about relevant degrees of freedom that can extend theoretical insights from theories of microscopic events to living systems? Science studies diverse time, length and energy scales 
and the gaps can be quite daunting. However if we look back from time to time, we will see how much progress has been made, so we do fill in gaps.

Chapter Two: Life

The second chapter, "Life”, has sections on "Energy and Entropy”, "Growth", "Reproduction and Death", and "Self-construction". This chapter discusses life from a thermodynamic perspective. After a brief consideration of entropy under a gravitational potential and fine tuning, the authors search for something universal in nature that can give us deeper insight into what is special about living beings. Rather than propose life's exceptionalism, the authors attempt to build bridges between thermodynamic and evolutionary categories, keeping track of where the shifting line between system and environment lies. The authors are not alone in this pursuit, and Creative Nature is in conversation with Stuart Kauffman and his proposals such as a 4th law of thermodynamics (Kauffman 2003, ix-xii).

The authors consider living beings in depth: their growth, reproduction, death and organization. Living beings are in a process of maturing and preparing for the future. Although we distinguish various stages of the life cycle, "maturation is the process that prepares [living beings] to do what will guarantee their future survival; and, as we have seen, the survival of living systems is guaranteed by reproduction" (Novo et al. 2018, 53).

Life acts on the environment and responds to the environment. Although living beings are always in a state of changing, they maintain the dynamic equilibrium of homeostasis precisely through a dynamic and complex molecular dance. Living beings change to avoid radical discontinuities. If the external stimulus (chemical, thermal, mechanical, electronic, etc.) is too dramatic then their integrity dissolves and the static equilibrium of biochemical death follows.

Upon closer inspection at a molecular level the tree of life has connections between distant parts, like a vine connecting distant branches. Beyond classification, the study of living beings strives to articulate precisely what is happening in the cycle of life. In the third section "Reproduction and death" the authors reflect on how living beings communicate information, 
which some authors have signalled out as the secret ingredient of life (De Loof 2017).

Reproduction allows the survival of the system in the very long run through copying itself. These copies have an independent existence and are obtained through the transmission of information related to the structure, internal dynamism, survival strategies and ways of reacting to external changes that are specific to each living system. Thus, a living being survives in time through the storage and transmission of information. Despite how incredibly complex this process seems, all the living natural systems on our planet are capable of storing and transmitting information with great efficiency. (Novo et al. 2018, 56-57)

In Creative Nature, life and death are contextualized in the life cycle, with past and future dependencies and constraints, rather than abstracting living from time and community and only considering an organism as a timeless solitary entity. Individuals come from prior life and are constrained to beget life, at least in enough of the population to ensure its persistence in history. Although many of us have an instinctual criteria for life as something creeping and crawling-we point and proclaim “It's alive!" à la Victor Frankenstein-the authors forge deeper into the mystery of life in ways that could perhaps enable us to recognize forms of life, in our own planet and beyond, even if they be biochemically diverse from life as we know it. ${ }^{10}$

In the proto-cell that we have just described, all the basic elements that are part of a living natural system are integrated: a program, a metabolism and a container. Effectively, the carrier molecules of instructions offers the program, the necessary information to maintain a certain identity in the set of all possibilities; chemical reactions generate the energy necessary to maintain the homeostasis of the system with its basic structures, and the membrane functions as a physical barrier that separates the system from the outside. With more or less complexity, this configuration is found in all forms of life that occur on Earth, and the same biomolecules fulfill in all of them the different roles of program, metabolism and container. Moreover, we can venture that if we were to find living natural

10 See The Harvard Origins of Life Initiative (Harvard 2018) and in particular a recent talk to see the current state of the field of astrobiology, 3rd Origins Prize Lecture (Seager 2018). 
systems in another place or time in the Universe, they probably have a similar configuration, even if the elements that make them up-the molecules-were different. (Novo et al. 2018, 62-63)

The last section of the chapter, entitled self-construction (Auto-construcción), is a term employed in a housing construction context to describe a do-it-yourself approach. A living being builds itself. The authors explore the analogy and where its applicability fades away in meaning. Although a living being receives initial information and starting material, the process of interior arrangement springs from its internal dynamics, and not from external hired hands. The authors caution the reader against simplistic mental imagery. A cell is not as it appears in a sleek, streamlined, textbook figure with 2D organelle islands floating on a sea of white paper. The dynamics of living beings transcend auto-assembly, although that concept applies to spontaneous associations at work among biomolecules. Self-organizing nonlinear systems such as Cells of Bernard / Rayleigh-Benard convection are present in non-living entities, and in life are subsumed into higher level layers of hierarchical complexity. As imaging technology advances we seem to be uncovering more and more crowding, complexity and information. In living beings, and communities, we find organization at various length and time scales where the parts contribute something for the sake of the whole (Dutta et al. 2018 and Burley et al. 2018). Living beings do not build themselves like we build a house, where there is a finished product that is separate and isolated from the activity of life. ${ }^{11}$ Life has many stages and a living being is alive during them all.

Living beings build a "whole," a complete dynamic system, through an informational process that guarantees homeostasis and the future possibility of reproduction. This process is known as development. This difference can be explained if we consider that the house is only a house when the self-assembly

11 See Aristotle, Nicomachean Ethics, I.1, 1094a3-5: "But a certain difference is found among ends; some are activities, others are products apart from the activities that produce them." 
process has been completed. The living being, on the contrary, is a living being from the beginning of its development process. (Novo et al. 2018, 65)

Experiments with sea sponges illustrate this point. If we cut a sponge into pieces they do not spontaneously assemble into a similar configuration, but each small piece grows into its own sponge. In fact, many living beings can reproduce without a mate, whether it is single cell yeast budding or a hydra budding off a parent to form a completely new entity. Typical of the style of Creative Nature, the authors flesh out general philosophical language with concrete examples, since their reflection is anything but pure a priori, but instead driven by empirical studies.

The section culminates with a definition of life, "a process of self-directed development that uses high energy and low entropy and dissipates low energy and high entropy, thus escaping, at least for a time, from the inexorable thermodynamic end that awaits inert matter."

Chapter Three: Change

Every material entity is something and can become something else. ${ }^{12}$ While the authors recognize that the material world we first experience with our senses is changing, this chapter considers only living beings. While reflection on other specialized topics would also interest many readers, the authors chose to concentrate on the history of life rather than the cosmos; biological networks instead of material science; the classification of living beings rather than subatomic particles.

Life is a chaotic system (Novo et al. 2018, 84). The authors link physics concepts from earlier chapters with recent experimental findings and research trends in biology, especially at a molecular level. The authors establish this link between physics and biology in three chapter sections: "Does everything change?" "Evolution and Genetics", "Macroevolution" and "Eco-Evo-Devo".

12 Even children from a tender young age, say 7 or 8 years old, are capable of understanding this definition (Rizzi 2012). 
The reader is led through a short historical journey of evolutionary thought and reminded that the view that the natural order in life arose over a historical time period is relatively new (Lamarck 1744 - 1822, Darwin 1804 - 1882, Mendel 1822 - 1884). This linear view was not shared by some ancient Greeks, such as Aristotle's school, who argued that the current state of affairs had always been what it was. However, as one medieval heir of the ancient Greeks observed, "The more long-lasting something is, the more time is needed so that its change might be discovered." ${ }^{13}$ The section on macroevolution gives specific details on microscopic mechanisms and biomolecules. They illustrate how changes in networks of microscopic relationships cause macroscopic observable changes.

They conclude the chapter by uniting three fields in science: evolutionary theory, the developmental process by which organisms grow and mature, and finally the ecological context of a living being with its surrounding environment (external and internal/microbiotic): Eco-Evo-Devo. ${ }^{14}$ Their vision of the process of becoming presents a rich and unified vision of change that makes progress from humble beginnings in the 19th century and the update of the modern synthesis in the 20th.

Today we know that evolution is the response to genetic, environmental and ecological changes mediated by the reconfiguration of genetic regulation networks that control the development programs of living beings. The structure and dynamics of these networks allow us to explain their plasticity and robustness. And at the same time, we have learned how some genetic alterations can modify the structure of these networks and thus lead to relatively important evolutionary changes in a short time. [...] Today we know that the metaphor for evolution of slowly climbing to the peak of the mountain, millimeter by millimeter, but always climbing, is erroneous. In fact, there does not have to be a single peak to climb. Nor is it clear exactly what it means to always go up. Rather, evolution flows as we walk through the park, moving through a landscape formed by hills and hollows, rising a few times, lowering others, and even jumping from

13 Aquinas 1886, De Caelo, lib. 1, lect. 7, n. 6, Leon., 3.

14 The authors use "Evo-Devo-Eco", which can also be found in the English literature along with "Eco-Evo-Devo", which we use here. 
one rock to another at times. Obviously, successful adaptive strategies will be different depending on whether we are facing a more or less orderly landscape or we are rather faced with a rough and rugged landscape, with many peaks and valleys that prevent us from passing from one place to another easily. (Novo et al. 2018, 99-100)

Chapter Four: Limits

Creative Nature's account of the limits and contours of the evolutionary change discussed in the third chapter is the topic of Chapter Four. It considers, in turn, "Adaptations," "Contingencies," and "Restrictions," in order to answer the question "What is it that natural selection really 'selects'?" (Novo et al. 2018, 123) In doing so, the authors shed light on how contemporary biology avoids the tautological answer that, over time, natural selection picks out the best adapted individuals, i.e., the surviving individuals. For, isn't this just the same as saying that the "those individuals survive that have survived?" (Novo et al. 2018, 124)

The authors had ended Chapter Three with the question about the limits of changes brought about by the process of evolution, and thus open the first section of Chapter Four, "Adaptations," by asking about the nature of the results of the adaptive or evolutive process. Are just any adaptations possible? Might it also be the case that some adaptations are frequently "discovered" through evolution as the necessary solutions to the demands of survival? Are some adaptations possible that don't necessarily add much to an organism's chances of survival? Examples like the platypus serve to help the reader's mind attend to the subsequent discussion of the possibility space or "evolutionary landscape" that captures in a mental model the contingencies and contours of the evolutive process.

The discussion of contingency in nature pits, on the one hand, a pure or total contingency against a contingency coexisting with what must of necessity be the case, on the other hand. They reject the former by appealing to what has been observed in nature. The evolutive process frequently "finds" the same adaptations, even in populations separated in time and space. 
The convergence and parallelism given by evolution clearly show that some biological solutions are more probable than others, and even permit one to assert that some are tremendously improbable. Still, it is difficult to be certain that some solution is totally impossible. This is an option that cannot be ruled out. (Novo et al. 2018, 109)

This convergence and parallelism permits one to understand, in part, the deep homologies in body plan or structure that are shared by many living beings. Evolution as a natural process is not characterized by a totalizing contingency that abhors all constancy or commonality among form. By contrast, some adaptations, especially those engineered in laboratory environments, are rare or even unique. In the end, as indicated in the quote above, our authors respect Nature's mutability: the evolutive process can still produce surprises. Nonetheless, the consistency within this contingency raises the question about how this contingency is and should be thought of as limited.

These restrictions on the contingencies of the evolutive process are discussed using Sewall Wright's metaphor of the adaptive landscape. The metaphor permits us to mentally inhabit the space of solutions sought out by evolution in such a way as to explain the peculiarities of some adaptations (e.g., the ones with no apparent adaptive value), the deep homologies in nature, and the stability of various forms of life over millions of years. In other words, the intrinsic combinatorial possibilities of genetic networks discussed in previous chapters, together with an interaction between a population of organisms and their surroundings (which environment imposes upon those individuals the contingently determined boundary conditions that define survival), yield the possible, and possibly changing or dynamic, contours of the adaptive landscape. This is the contingency within limitation that permits the evolution of adapted forms of life.

The commonly accepted perspective is that in each living system there is a series of levels or formal resources (types of networks) responsible for maintaining the identity of the system, while there are other levels or resources that are used to explore new adaptive possibilities, optimizing strategies without compromising the global unity of the system. This sort of function seems to be a formal law 
of complex systems, that is, a law that is independent of the concrete material from which they are constituted. The stability and plasticity of living beings, likewise, seems to be optimized in evolution. (Novo et al. 2018, 121-122)

Indeed, this common viewpoint, insofar as it makes an appeal to a "formal law" independent of particular environmental niches, does not rule out the possibility of similarly evolved forms of life in different galaxies, yet neither does it demand it (Novo et al. 2018, 122-123).

This discussion permits the authors to offer a defining characteristic of life, namely, evolvability. A living thing must be something capable of evolution.

As we have seen, the fact that living systems are constituted by modular networks is the basis both for the plasticity they exhibit as well as the stability or resilience also necessary for evolution to exist. That is, in the final analysis, what natural selection really 'selects' is precisely the capacity to evolve. Put in other terms, evolvability is the property necessary for all living beings. The concept of evolvability suggests, furthermore, a type of change that does not compromise the identity of the system. Living beings always maintain a space of possible changes within their internal organization so as to adjust themselves to the ups and downs of an environment that cannot be foreseen in its totality. They must be prepared to change, anticipating even changes in the environment, if they want to stay alive. Without this property, the capacity to preserve identity through change, life could not have prospered. Our planet would be one more amidst the inert systems of the universe. (Novo et al. 2018, 125-126)

This leads to another defining feature of life proposed in Chapter Five: survival.

Chapter Five: Functions

Our authors approach the topics of function and goal-seeking in biology in three sections: "Causes and Reasons," "Form or Function?," and "Direction and Order." Their discussion serves to substantiate the conclusion that "the finality and immanent order that we discover upon observing the reality that surrounds us is in nature itself" (Novo et al. 2018, 148). Nature's order 
or finality, especially in the biosphere, "is no projection, nor does it arise by the imposition of any manner of external agent." This intrinsic finality combining the genetic and environmental causes of evolvability with the end of survival brings to mind Aristotle's reason for reproduction as the hallmark of life. He maintains that each species of living thing reproduces

in order that, as far as its nature allows, it may partake in the eternal and divine. That is the goal towards which all things strive, that for the sake of which they do whatsoever their nature renders possible. [...] Since then no living thing is able to partake in what is eternal and divine by uninterrupted continuance [...], it tries to achieve that end in the only way possible to it, and success is possible in varying degrees; so it remains not indeed as the self-same individual but continues its existence in something like itself-not numerically but specifically one. (Aristotle, On the Soul, II.4, 415a29-415b7)

What we moderns change of Aristotle's view, of course, is the means by which nature as an originative cause accomplishes the end of survival. No longer do species remain eternally fixed, but are subjected to change over eons. What does not change is the claim that nature imbues living systems with an intrinsic order to the survival of living things at a supra-individual level.

Evolution and adaptation, within the limits and possibilities imposed by the processes of development, would therefore be the fundamental and foundational properties of the living. If someone asked us what is the finality of living things, we could respond unambiguously: in an ever-changing ecological context, the finality is to survive. Life perpetuates itself by diversifying itself. (Novo et al. 2018, 149)

We should examine how and to what extent our authors reinstate this finality within nature.

Their first step, in the section "Causes and Reasons," is to qualify the extent to which mechanistic explanations can successfully account for nature. By "mechanism" they mean "the concrete disposition-spatial and temporal-of a set of elements that form part of a system, along with the interactions between each one of those elements" (Novo et al. 2018, 128). 
They do not deny the success of mechanistic explanation within its proper ambit. Now, on the one hand, this notion of mechanism uses a notion of causality defined by temporal succession, or a diachronic connection between cause and effect. Our authors therefore countenance the possibility that causes or reasons can be synchronic, or temporally coincident with each other, enduring over a span of time. On the other hand, current discussions of causality identify the necessary elements for a causal explanation with being able to provide the necessary and sufficient conditions for some event or feature of a thing. However, due to the complexity of natural events as well as difficulties of scale, it is frequently too difficult to satisfy this criterion. They therefore begin to search for a broader notion of causality and explanation.

This leads them to the notion of "function":

In general, this term is more abstract than mechanism, designating something that is broader and, consequently, more difficult to specify. The function of something is that for which it exists, that for which it is useful. It is a meaning that is very close to the reason why something is thus and not otherwise [...] If we adopt the term "function," it is to avoid the error of explaining everything as mere mechanism. The study of a system permits one to observe that each part of this whole, together the mechanisms in which they partake, fulfill a function. That is, the parts of a system are there for something, they are useful for something, then have an objective, a certain finality within the whole that constitutes the system. (Novo et al. 2018, 131-132)

In turn, they examine possible "functions" of even inert things (the "function" of an earthquake), just to illustrate the ubiquity of this mode of thinking. However, the discussion narrows from this broad sense to the debate, which they begin with Lamarck, about the priority of form or function. They note the strong tendency to anthropomorphize organic functionality in living things; surely there is a need to pump blood, and thus the function or purpose of pumping blood demands an organ of a certain shape and structure. However, they argue that the course of events went in the reverse: certain structures had various potential functions and thus, over 
time, determinate functions were selected in or selected out. They take the penguin's wings as an example. Consequently, they posit an evolutionary definition of function: "The function of any structure is that for which evolution has selected it; the evolutionary 'reason' due to which this structure possesses such a form" (Novo et al. 2018, 136). It is by such means that our authors dissolve the debate over the priority of form and function within the evolutionary process they have been discussing throughout the book. The problem of priority is only an apparent one, they say, insofar as the set of possible forms known through developmental biology indicate the potential functions of an organ whose true purposes have been determined through adaptive selection:

From this perspective, the problem of the primacy of form or function is only apparent, since the set of possible forms (which we know thanks to developmental biology) tells us what functions an organ can perform. In turn, the effectiveness with which that organ performs certain functions will establish, through natural selection, its evolutionary success and dictate which variants will ultimately prevail. Therefore, we cannot correctly understand the function that an organ or structure performs in this particular species and in this particular ecological context without attending to the functions it has performed throughout its evolutionary history. In turn, these functions will have varied depending on the different morphological configurations (forms) through which it has passed. (Novo et al. 2018, 138-139)

This then raises the question of whether or not the evolutionary process itself possesses an order or finality. Again, they helpfully note how the language of purposive directionality is even embedded in description of simple local motion, or in descriptions of the abstract adaptive landscape. However, is such talk of purpose mere talk or does it latch onto something in reality? Indeed, they recall that evolution exhibits both plasticity and a certain robustness. The evolutive process is non-ergodic ${ }^{15}$ in certain key

15 Stuart A. Kauffman explains: "Non-ergodicity stands in contrast to 'ergodicity.' 'Ergodic' means that the system in question visits all its possible states. In Statistical Mechanics this is based on the famous "ergodic hypothesis, which, mathematically, gives up inte- 
respects; it is not entirely random. Yet does it have finality? Our authors say yes (as indicated just above), and their discussion merits a closer inspection, which we undertake in $\$ 3.2$, below.

\section{Chapter Six: Creativity}

The final chapter considers, in turn, "Laws" and "Emergence" in nature. The discussion is a fitting capstone for the themes discussed in the book, since it takes the broad view, allowing the reader to recollect in a single whole all the various details. Their question: Can the world be explained by deterministic laws? Our authors, as might be expected given their previous arguments, answer "No.”

We have addressed the question whether some day the physical world-from the microscopic to the biological-might be completely explained by deterministic laws. Various advances in science during the last few decades indicate that this will not be possible. (Novo et al. 2018, 178)

They give three examples: quantum non-locality, the degrees of freedom enjoyed by physical systems, and, finally, biological indeterminacy:

This intrinsic indetermination of the physical world is even clearer in biology, where one truly cannot speak of laws in the proper sense, but rather of functional models and ideal conditions. (Novo et al. 2018, 179)

In this spirit, the discussion of the chapter progresses from the question of the possibility of determinism to its demise in view of advances in the sciences, from physics to biology. Following Kant's saying, that there can be no Newton for a blade of grass, ${ }^{16}$ they gradually introduce the reader to the

gration of Newton's equations of motion for the system. Ergodic systems have no deep sense of "history.' [...] The evolution of life in our biosphere is profoundly "non-ergodic" and historical. The universe will not create all possible life forms. This, together with heritable variation, is the substantial basis for Darwin, without yet specifying the means of heritable variation, whose basis Darwin did not know. Non-ergodicity gives us history.'” (Kauffman 2017).

16 "For it is quite certain that in terms of merely mechanical principles of nature we cannot even adequately become familiar with, much less explain, organized beings and how 
notion of emergence as superior to the "supervenience" of macrostates upon microstates (Novo et al. 2018, 167-168). This leads them to discuss Terence Deacon's three types of emergence, as well as broaching the possibility of top-down causality, as promoted by Nancy Murphy or George Ellis, among others. (For more on these themes, see \$3.2, below.) In this way, emergence and top-down causality add the finishing touches to a wide-ranging discussion of nature as essentially productive of order in the universe, but productive in a fashion that is open to innovation of order:

Given the nature of evolutionary processes, those in which the conditions of the environment are changing and create new possibility spaces, the future directions of change cannot be foreseen using some algorithm. [...] All of this brings us to the conclusion that nature possesses the characteristics of a process that is capable of realizing authentically emergent phenomena. This means that it is endowed with the creativity necessary to guarantee a future full of novelties, each one more beautiful and wonderful. (Novo et al. 2018, 179)

Our authors conclude their reflections with their belief, grounded on the natural creativity present in the universe, in the "open future" of the universe. This is a moral corollary to the scientific wonder about nature sustained through the volume. Their attitude is not that of the "master and possessor of nature” envisioned by Francis Bacon's New Atlantis or Rene Descartes's Discourse on modern scientific method. Rather, they exhort the reader to a collaborative, human project of knowledge and hope.

\section{Pedagogical Possibilities in Conclusion}

As noted in the introduction, a co-led, interdisciplinary seminar would be a fitting context to work through and learn from this monograph. Alterna-

they are internally possible. So certain is this that we may boldly state that it is absurd for human beings even to attempt it, or to hope that perhaps some day another Newton might arise who would explain to us, in terms of natural laws unordered by any intention, how even a mere blade of grass is produced. Rather, we must absolutely deny that human beings have such insight” (Kant 1987, §75, 282-83). 
tively, an extra-curricular reading group composed of a mix of philosophy and science majors would profit greatly from working through this text. The emphasis we wish to place is on taking advantage of the book's interdisciplinary approach, as we have attempted to underline in our summary and "key points". Creative Nature is not a work intended to be read from within one's academic specialty, but one intended to be discussed with others inside and outside our university department offices.

In the next part of this review essay, we will develop various points for deeper reflection for such seminars or discussions.

\section{References}

Artigas, Mariano. 2006. Knowing Things for Sure: Science and Truth. Lanham, MD: University Press of America.

Artigas, Mariano. 2001. The Mind of the Universe: Understanding Science and Religion. West Conshohocken, PA: Templeton Foundation Press.

Aquinas, St. Thomas. 1918. Opera Omnia Iussu Leonis XIII P. M. Edita, t. 13: Summa Contra Gentiles Liber 1 \& 2 Cum Commentariis Ferrariensis. Vol. 13. 50 vols. Romae: Ex Typographia Polyglotta S. C. de Propaganda Fide.

Aristotle. 2014. The Complete Works of Aristotle: The Revised Oxford Translation, One-Volume Digital Edition. Edited by Jonathan Barnes. Princeton, NJ: Princeton University Press.

Ashley, Benedict M. 2006. The Way toward Wisdom: An Interdisciplinary and Intercultural Introduction to Metaphysics, Thomistic Studies. Notre Dame, IN: University of Notre Dame Press.

Bershtein, Shimon, Serohijos, Adrian W.R., and Eugene I. Shakhnovich. 2017. "Bridging the physical scales in evolutionary biology: From protein sequence space to fitness of organisms and populations." Curr. Opin. Struct. Biol. 42: 31-40.

Burley, Stephen K., Berman, Helen M., Christie, Cole, Duarte, Jose M., Feng, Zukang, Westbrook, John, Young, Jasmine, and Christine Zardecki. 2018. "RCSB Protein Data Bank: Sustaining a living digital data resource that enables breakthroughs in scientific research and biomedical education." Protein Science 27(1):316-330. http://doi.org/10.1002/pro.3331

De Loof, Arnold. 2017. The evolution of "Life": A Metadarwinian integrative approach. Communicative and Integrative Biology 10(3):e1301335. Accessed 26 June 2018. http://doi.org/10.1080/19420889.2017.1301335 
Dutta, Shuchismita, Eswaran, Subha, Sanelli, Anne, Bhattacharya, Meenakshi, and Richard Tempsick. 2018. "Learning biology through molecular storytelling." The Science Teacher 86: 28-33.

Ellis, George F. R. 2016. How Can Physics Underlie the Mind?: Top-Down Causation in the Human Context. Berlin/Heidelberg: Springer.

Harvard University. 2018. “Origins of Life Initiative.” Accessed 15 Aug 2018. https:// origins.harvard.edu/

Kant, Immanuel. 1987. Critique of Judgment. Translated by W. S. Pluhar. Indianapolis, IN: Hackett Publishing.

Kauffman, Stuart A. 2003. Preface to Investigations. Oxford: Oxford University Press. Murphy, Nancy and William. R. Stoeger, ed. 2007. Evolution and Emergence: Systems, Organism, Persons. Oxford: Oxford University Press.

Murphy, Nancy and Warren S. Brown. 2007. Did My Neurons Make Me Do It?: Philosophical and Neurobiological Perspectives on Moral Responsibility and Free Will. Oxford: Oxford University Press.

Novo, Javier, Pereda, Rubén and Javier Sánchez-Cañizares. 2018. Naturaleza Creativa. Madrid: Rialp.

Origins of Life Initiative. 2018. "Video of 3rd Origins Prize Lecture: Sara Seager (MIT) 'Exoplanets and the Search for Biosignature Gases: An Astronomer's Journey through Chemical Space', 19 September 2018." https://origins.harvard.edu/news/ video-3rd-origins-prize-lecture-sara-seager-mit-exoplanets-and-search-biosignature

Rizzi, Anthony. 2018. Physics for Realists: Quantum Mechanics. Modern Physics with a Common Sense Grounding. Baton Rouge, LA: IAP Press.

Rizzi, Anthony. 2012. “What is a Physical Thing?” In A Kid's Introduction to Physics (and Beyond), 9-12. Baton Rouge, LA: IAP Press.

Sacks, Jonathan. 2012. The Great Partnership: Science, Religion, and the Search for Meaning. New York, NY: Schocken.

Unger, Roberto Mangabeira and Lee Smolin. 2014. The Singular Universe and the Reality of Time: A Proposal in Natural Philosophy. Cambridge: Cambridge University Press. 